\title{
Macrophages Protect against Muscle Atrophy and Promote Muscle Recovery in Vivo and in Vitro
}

\author{
A Mechanism Partly Dependent on the Insulin-Like Growth \\ Factor-1 Signaling Molecule
}

\author{
Nicolas Dumont, ${ }^{*}$ and Jérôme Frenette ${ }^{\star \dagger}$ \\ From the Centre Hospitalier Universitaire de Québec-Centre de \\ Recherche du Centre Hospitalier de l'Université Laval," and \\ Département de Réadaptation, ${ }^{\dagger}$ Faculté de Médecine, Université \\ Laval, Quebec City, Quebec, Canada
}

\begin{abstract}
Hindlimb unloading and reloading are characterized by a major loss of muscle force and are associated with classic leukocyte infiltration during recovery from muscle atrophy. Macrophages act as a cellular cornerstone by playing both pro- and anti-inflammatory roles during muscle recovery from atrophy. In the present study, we investigated the role of macrophages in muscle atrophy and regrowth using in vivo and in vitro models. Mice depleted in monocytes/ macrophages and submitted to a hindlimb unloading and reloading protocol experienced a significant delay in muscle force recovery compared with matched placebo mice at 7 and 14 days after reloading. Furthermore, an in vitro myotube/macrophage coculture showed that anti-inflammatory macrophages, which contain apoptotic neutrophils and express low levels of cyclooxygenase- 2 , completely prevented the loss of protein content and the myotube atrophy observed after 2 days in low serum medium. The presence of macrophages also protected against the decrease in myosin heavy chain content in myotubes exposed to low serum medium for 1 day. Interestingly, the addition of an anti-IGF-1 antibody to the coculture significantly decreased the ability of macrophages to protect against myotube atrophy and myosin heavy chain loss after 2 days in low serum medium. These results clearly indicate that macrophages and, more precisely, the release of IGF-1 by macrophages, play an important role in recovery from muscle atrophy. (Am J Pathol 2010, 176:2228-2235; DOI: 10.2353/ajpath.2010.090884)
\end{abstract}

Monocytes are large mononuclear cells that circulate in the blood and differentiate into macrophages in invaded tissues in response to various stimuli. ${ }^{1}$ Macrophages have a strong phagocytic capacity and can orchestrate the inflammatory process via the release of a wide variety of cytokines and chemokines such as interleukin (IL)-1, tumor necrosis factor- $\alpha$, and macrophage inflammatory protein-2. ${ }^{2,3}$ Numerous studies have also demonstrated that macrophages play a direct role in tissue recovery through the release of the anti-inflammatory molecules and anabolic growth factors IL-10, basic fibroblast growth factor, and insulin-like growth factor-1 (IGF-1). ${ }^{4-6}$

The regulation of the multiple and occasionally opposing functions of macrophages is very complex and poorly understood. $^{7-9}$ To add to this complexity, the diversity of experimental models can also lead to different conclusions regarding the roles of each macrophage phenotype in muscle recovery from atrophy or damage. For example, in a model of eccentric contractions, in which injured muscle is basically devoid of neutrophils, macrophage invasion contributes to secondary damage to the skeletal muscle. ${ }^{10}$ On the other hand, the phagocytosis of apoptotic neutrophils and necrotic cells by macrophages induces a change in macrophage phenotype from pro- to anti-inflammatory, which has a strong modulatory effect on cytokine profiles and is essential for dampening environmental inflammatory signals. ${ }^{11,12}$ Regarding the different subsets of macrophages, coculture experiments have shown that pro-inflammatory macrophages (Ly$\left.6 \mathrm{C}^{\text {hi }}\right)$ stimulate myogenic cell proliferation, whereas anti-inflammatory macrophages (Ly-6Clo $)$ exert a strong differentiating influence on myogenic cells. ${ }^{12,13}$ Nonethe-

Supported by grants from the Canadian Institutes of Health Research and the Natural Sciences and Engineering Research Council of Canada.

Accepted for publication December 29, 2009.

Address reprint requests to Jérôme Frenette, P.T., Ph.D., CHUQCRCHUL, 2705 Boulevard Laurier, T-R-93, Quebec City, QC, Canada G1V 4G2. E-mail: jerome.frenette@crchul.ulaval.ca. 
less, in a model in which rodent hindlimbs are deprived of mechanical loading for 10 days followed by reloading, the high concentration of leukocytes in reloaded muscles regardless of the absence of significant muscle damage raises intriguing questions about the detrimental and beneficial roles of leukocytes in muscle dysfunction and recovery from atrophy. ${ }^{14-16}$ It is thus tempting to speculate that the roles of macrophages may vary as a function of the type of insult.

In the present study, mice depleted in macrophages were submitted to hindlimb unloading and reloading to evaluate the roles of macrophages in muscle atrophy and regrowth. Our results showed that macrophages neither prevent the loss in muscle force nor promote recovery during the early inflammatory phase ( 1 and 3 days after reloading). However, they play a key role in muscle growth and recovery at later times ( 7 and 14 days after reloading). In addition, an in vitro coculture model in which atrophied myotubes were combined with macrophages expressing an anti-inflammatory phenotype showed that the presence of macrophages protects myotubes from atrophy and that this protective effect is partly mediated by the release of IGF-1.

\section{Materials and Methods}

\section{Animals}

Male C57BL/6 mice (22 to $24 \mathrm{~g}$ ) from Charles River (St-Constant, QC, Canada) were given water and food ad libitum and housed with a 12-hour light/dark cycle. Experimental mice were subjected to hindlimb unloading for 10 days using a modification of the technique developed by Morey-Holton and Globus. ${ }^{17}$ The hindlimbs were then reloaded for $0,3,7$, or 14 days. Ambulatory mice were used as controls. Mice were euthanized by cervical dislocation under anesthesia. All procedures were approved by the Université Laval Research Center Animal Care and Use Committee based on Canadian Council on Animal Care guidelines.

\section{Monocyte/Macrophage Depletion}

To determine the specific roles of monocytes/macrophages in muscle recovery from atrophy, etoposide (VP16, Sigma, St. Louis, MO) diluted in dimethyl sulfoxide (15 $\mathrm{mg} / \mathrm{kg}$ of mouse mass) was injected daily for 3 days before reloading until the end of the experimental protocol. Etoposide acts as an inhibitor of topoisomerase II and is used in the treatment of some malignancies such as nonlymphocytic leukemia to inhibit white blood cell proliferation. ${ }^{18}$ Placebo mice received daily injections of dimethyl sulfoxide. The effects of etoposide on leukocyte counts were confirmed by flow cytometry as described previously. ${ }^{14}$ In brief, blood was collected by cardiac puncture, incubated with blocking rat IgG (Sigma), and labeled with $0.2 \mathrm{mg}$ of rat anti-mouse Ly-6G R-phycoerythrin-conjugated antibody or $0.4 \mathrm{mg}$ of rat anti-mouse F4/80 R-phycoerythrin-conjugated antibody (primary antibodies, BD Pharmingen, Franklin Lakes, $\mathrm{NJ}$ ) to detect neutrophils and macrophages, respectively. R-phycoerythrin-conjugated rat anti-mouse IgG was used as an isotype control antibody at the same concentrations (BD Pharmingen). Flow cytometry was performed using an EPICS XL flow cytometer (Beckman-Coulter, Fullerton, CA).

\section{Isometric Contractile Properties}

Mice were injected i.p. with buprenorphine $(0.1 \mathrm{mg} / \mathrm{kg})$ as an analgesic and anesthetized with pentobarbital sodium (50 mg/kg) 15 minutes later. ${ }^{19}$ Right soleus muscles were carefully dissected, attached to an electrode, and incubated in a buffered physiological salt solution (Krebs-Ringer) supplemented with glucose $(2 \mathrm{mg} / \mathrm{ml}$ ) for absolute and specific muscle force measurements as described previously. ${ }^{14,20}$ Absolute muscle force represents the total force generated by the muscle, whereas specific muscle force also accounts for muscle mass and fiber length. Thereafter, muscle length was measured, tendons were removed, and the muscles were weighed. The muscles were then embedded in tissue freezing medium (Triangle Biomedical Sciences, Durham, NC), frozen in isopentane (2-methylbutane, Sigma) cooled in liquid nitrogen, and stored at $-80^{\circ} \mathrm{C}$ until used.

\section{Immunohistochemistry and Cell Counting}

Transversal sections (10 $\mu \mathrm{m}$ ) of soleus muscles were cut (CM1850 cryostat, Leica Microsystems, Nussloch, Germany) at $-20^{\circ} \mathrm{C}$ and adhered to Snowcoat X-tra slides (Surgipath, Richmond, IL). After 1 hour in a blocking buffer, the sections were incubated for 2 hours with rat anti-mouse F4/80 antibody (Serotec, Oxford, UK) to identify macrophages or rat anti-mouse Ly-6G/Ly-6C antibody (RB6-8C5, BD Pharmingen) to identify neutrophils. The sections were then incubated with rabbit anti-rat biotinylated IgG (secondary antibody, Vector Laboratories, Burlingame, CA) for 1 hour. Lastly, the sections were incubated for 30 minutes with horseradish peroxidase avidin D (Vector Laboratories) and revealed using diaminobenzidine chromogen (DakoCytomation, Carpinteria, CA). Each section was examined by light microscopy at a magnification of $\times 400$, and the number of labeled cells in the entire section was determined and expressed per $\mathrm{mm}^{2}$. The number of inflammatory cells was measured in duplicate on two mid-belly sections from both the left and right soleus muscles. The averages of four sections per mouse per antibody were used for the statistical analyses.

\section{Cell Culture and Myotube Atrophy}

C2C12 mouse myoblasts and J774 macrophages (American Type Culture Collection, Manassas, VA) were used for the in vitro experiments. C2C12 myoblasts were used for their ability to form rapidly contractile myotubes, whereas $\mathbf{J 7 7 4}$ macrophages exhibit predominantly antibody-dependent phagocytosis capacity and, more importantly, are known to inhibit the release of proinflammatory mediators and stimulate the secretion of anti-inflamma- 
tory molecules after the phagocytosis of apoptotic cells. ${ }^{21-23}$ Cells were grown in high-glucose Dulbecco's modified Eagle's medium (HyClone, Waltham, MA) supplemented with 10\% fetal bovine serum (Sigma) and 1\% antibiotic-antimycotic (Gibco, North Andover, MA) in 5\% $\mathrm{CO}_{2}$ at $37^{\circ} \mathrm{C}$. The medium was replaced every 3 days, and the cells were used for a maximum of 10 passages. C2C12 myoblasts were seeded in six-well plates at a density of 25,000 cells $/ \mathrm{cm}^{2}$ and incubated for 24 to 48 hours. When the myoblasts reached confluence, the medium was replaced by Dulbecco's modified Eagle's medium containing 2\% horse serum (Sigma) and 1\% antibiotic-antimycotic, and the confluent cultures were incubated for a further 5 days to allow differentiation into myotubes. Thereafter, myotubes were grown in Dulbecco's modified Eagle's medium containing 10\% of fetal bovine serum for 2 days. Myotubes were then large and well differentiated with relatively slow growing potential. Finally, to induce myotube atrophy, large myotubes were put in low-density serum medium (2\% horse serum) for 2 days.

\section{Macrophage/Myotube Cocultures}

A coculture system was developed to more precisely identify the effects of macrophages on muscle recovery from atrophy. Macrophages were first grown in the presence of apoptotic neutrophils, which triggers their conversion from a pro- to an anti-inflammatory phenotype. ${ }^{11,21}$ To isolate neutrophils, blood was collected by cardiac puncture and placed in 3\% Dextran 500 (GE Healthcare, Waukesha, WI) to sediment the red blood cells. The blood was centrifuged, and the pellet was suspended in $40 \mathrm{ml}$ of $0.15 \mathrm{~mol} / \mathrm{L} \mathrm{NaCl}$. Ficoll-Paque (10 $\mathrm{ml}$ ) (GE Healthcare) was carefully layered at the bottom of the tube, which was centrifuged for 40 minutes at $400 \times g$. The supernatant was discarded, and the remaining red blood cells were lysed in sterile water for no more than 30 seconds. The remaining cells were suspended in Dulbecco's modified Eagle's medium containing $10 \%$ fetal bovine serum. Flow cytometry revealed that $90 \%$ of the cells were neutrophils. The neutrophils were exposed to UV irradiation (254 $\mathrm{nm}$ ) for 15 minutes to induce apoptosis. The apoptotic neutrophils were cocultured with macrophages (2:1 ratio) for 3 hours. Neutrophils that had not been ingested by macrophages were removed by rinsing with PBS, whereas macrophages containing apoptotic neutrophils were added to myotubes at the beginning of the low serum medium phase (25 macrophages $/ \mathrm{mm}^{2}$ ). The ratio of apoptotic neutrophils/macrophages (2:1) and the concentration of macrophages in the coculture with myotubes were chosen because they mimicked in vivo conditions and provided reproducible results with atrophic myotubes. To analyze the influence of apoptotic neutrophils on macrophages, macrophages alone were used as a negative control and macrophages incubated with lipopolysaccharide (200 $\mathrm{ng} / \mathrm{ml}$, Sigma) were used as a positive control. Finally, to verify the role of IGF-1 in the macrophage-induced protective effect against myotube atrophy, an anti-mouse
IGF-1 antibody (5 $\mu \mathrm{g} / \mathrm{ml}$, R\&D Systems, Minneapolis, MN) was also added to the coculture system every 12 hours.

\section{Myotube Diameter Analysis}

Myotube diameters were measured by light microscopy at $\times 100$ (Nikon, Tokyo, Japan). Three different sites in each well were blindly identified and observed throughout the experiment. Myotube diameters were quantified using the ImageJ digital imaging system (National Institutes of Health, Bethesda, MD). For each myotube, a first measurement of the width was obtained $50 \mu \mathrm{m}$ away from the edge of the myotube and at every $200 \mu \mathrm{m}$ subsequently. This method usually allowed the recording of two or three measurements for each myotube, and the average was considered as a single value. The diameters of 150 to 200 myotubes per well were measured.

\section{Protein Content and Western Blots}

The myotubes were washed twice with PBS, incubated for 30 minutes at $4^{\circ} \mathrm{C}$ in a lysis buffer containing P8340 protease inhibitor cocktail $(1 \mu \mathrm{l} / \mathrm{ml}$, Sigma), and centrifuged for 10 minutes at 10,000 $\times \mathrm{g}$. The protein content of the homogenates was measured using BCA Protein Assay Kits (EMD Chemical, Darmstadt, Germany). In brief, $12,5 \mu \mathrm{l}$ of homogenate was added to duplicate wells of 96 -well plates followed by $200 \mu$ l of BCA solution. The absorbance was measured at $562 \mathrm{~nm}$ and compared with a standard curve. To accurately compare the protein content of the cultured myotubes incubated with or without macrophages, the protein content of the macrophages alone was determined and subtracted from the total to avoid overestimatation of the protein content of the myotubes in the coculture system. For the Western blot analyses, $10 \mu \mathrm{g}$ of protein were electrophoretically separated on a 9\% SDS-polyacrylamide gel and transferred to a polyvinylidene difluoride membrane. The membrane was blocked for 1 hour in 5\% skim milk and then incubated overnight at $4^{\circ} \mathrm{C}$ with anti-myosin heavy chain antibody (1:1000, Santa Cruz Biotechnology, Santa Cruz, CA) for myotube monocultures and cocultures or anti-cyclooxygenase-2 (COX-2) antibody (1:1000, Santa Cruz Biotechnology) for macrophage cultures. The membranes were rinsed and incubated with goat anti-rabbit (for myosin heavy chain, 1:10,000, Santa Cruz) or donkey anti-goat (for COX-2, 1:10,000, Santa Cruz Biotechnology) horseradish peroxidase-conjugated secondary antibodies diluted in 5\% skim milk. Bands were revealed using the ECL-Plus chemiluminescent detection system (PerkinElmer Life and Analytical Sciences, Wellesley, MA). Images of the membranes were acquired, scanned, and analyzed using Quantity One software (version 4.6.6, BioRad Laboratories, Hercules, CA).

\section{Statistical Analyses}

All values are reported as means \pm SEM. The data were analyzed by one-way analysis of variance to determine 
A



B

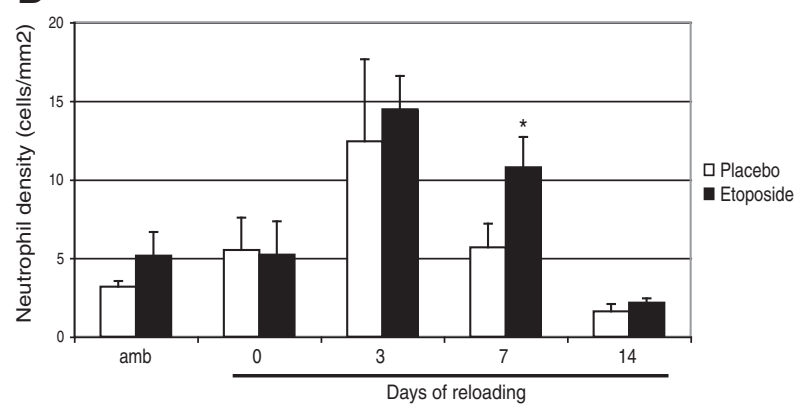

Figure 1. Macrophage and neutrophil concentrations in control and reloaded soleus muscles. Concentrations of macrophages (A) and neutrophils (B) in soleus muscles from mouse hindlimbs that were unloaded for 10 days and then reloaded for $0,3,7$, or 14 days. Ambulatory mice were used as controls. The mice received daily injections of etoposide or dimethyl sulfoxide beginning 3 days before the reloading until the end of the experiment. Values are expressed as means $\pm \operatorname{SEM}(n=6)$ for every group at every time point. Significantly different from reloaded placebo mice, ${ }^{*} P<0.05$.

whether the variations among the experimental groups were significant (JMP). When a significant $F$ ratio was obtained, a post hoc multiple comparison was performed using Tukey's protected least-significant differences test to determine whether specific differences had occurred. The level of significance was set at $P<0.05$.

\section{Results}

\section{In Vivo Model of Mice Hindlimb Unloading and Reloading}

Ten days of hindlimb unloading followed by 3 days of reloading increased the concentrations of macrophages and neutrophils in skeletal muscles by 10- and 4-fold, respectively. Daily injection of etoposide for 5 days reduced blood monocyte counts by $80 \%$ and circulating neutrophil counts by $20 \%$ (data not shown). Immunohistological observations of soleus muscles revealed that the number of macrophages in etoposide-injected mice had decreased by $70 \%$ by day 3 after reloading (Figure $1 \mathrm{~A})$. However, the number of macrophages in the soleus muscles was comparable with that in placebo mice on day 7 after reloading. Although etoposide decreased the number of circulating neutrophils slightly, the number of infiltrated neutrophils did not change during the various reloading periods, except at day 7 when a 2 -fold increase was observed in the reloaded etoposide group compared with the reloaded placebo group (Figure 1B).
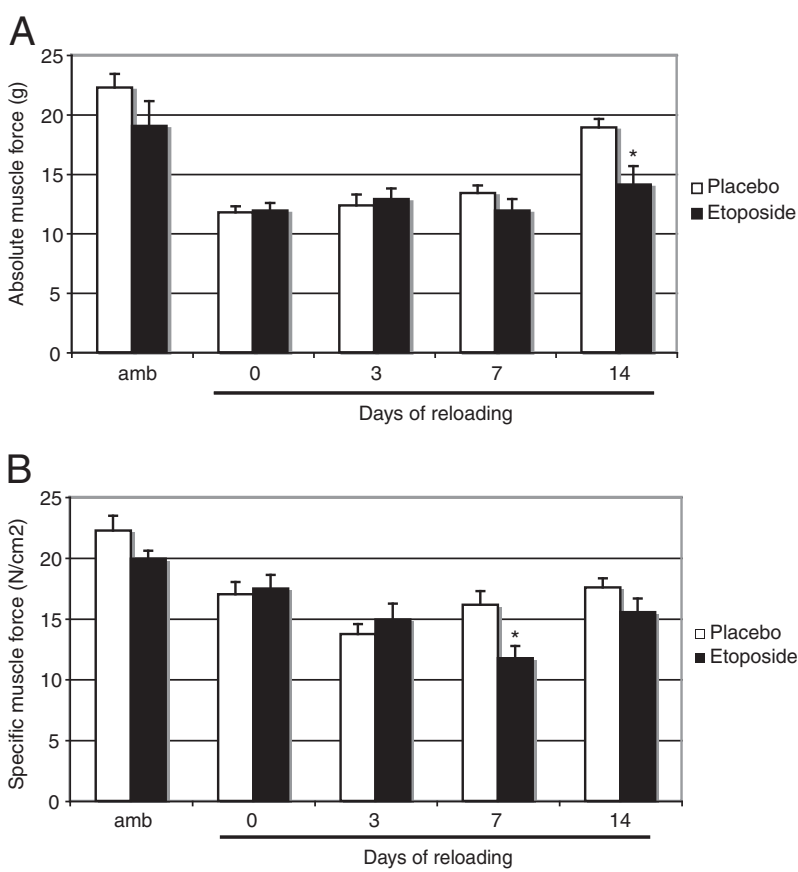

Figure 2. Absolute and specific muscle force production in control and reloaded soleus muscles. Absolute muscle force (A) and specific muscle force (B) were measured in soleus muscles from mouse hindlimbs that were unloaded for 10 days and then reloaded for $0,3,7$, or 14 days. Ambulatory mice were used as controls. The mice received daily injections of etoposide or dimethyl sulfoxide beginning 3 days before the reloading until the end of the experiment. Values are expressed as means $\pm \operatorname{SEM}(n=6)$ for every group at every time point. Significantly different from reloaded placebo mice, ${ }^{*} P<0.05$.

Consistent with previous observations, hindlimb unloading for 10 days caused a 50\% reduction in absolute muscle force, whereas specific muscle force was reduced by $25 \%$ relative to that in ambulatory mice. The etoposide-induced reduction in muscle macrophage content did not affect absolute muscle force on days 0,3 , and 7 after reloading, but considerably disrupted recovery on day 14 . Indeed, the average absolute muscle force generated by soleus muscles at day 14 of reloading is $25 \%$ lower in the macrophage-depleted mice than in placebo mice (average of 14 and $19 \mathrm{~g}$, respectively) (Figure 2A). A similar reduction was observed after 7 days of reloading, with the specific muscle force of reloaded macrophage-depleted mice being almost 30\% lower than that of reloaded placebo mice (average of 11.5 and $16 \mathrm{~N} / \mathrm{cm}^{2}$, respectively) (Figure 2B).

\section{In Vitro Model of Myotube Atrophy and Regrowth}

A coculture model was developed to further investigate the role of macrophages in recovery from muscle atrophy. The macrophage phenotype was determined by examining the levels of COX-2, a marker of inflammation. ${ }^{24-26}$ Western blot analyses clearly revealed a significant reduction in COX-2 in macrophages cocultured with apoptotic neutrophils compared with macrophages alone or macrophages incubated with lipopolysaccharide, indicating that the macrophage phenotype changed in the 


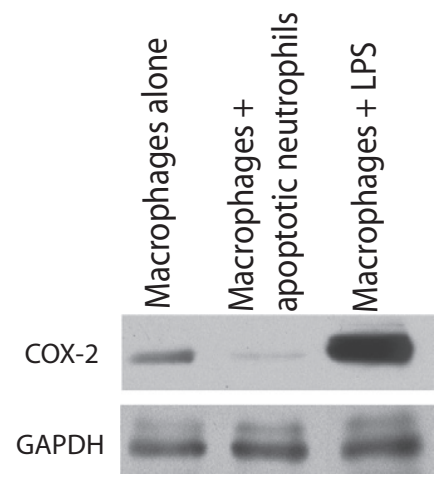

Figure 3. Identification of macrophage phenotype using COX-2 protein expression. The level of COX-2 protein was determined by Western blotting of proteins extracted from macrophages alone (negative control), macrophages containing apoptotic neutrophils, and macrophages incubated with lipopolysaccharide (LPS; $200 \mathrm{ng} / \mathrm{ml}$ ) (positive control). The COX-2 band has a molecular mass of approximately $72 \mathrm{kDa}$. Glyceraldehyde-3-phosphate dehydrogenase (GAPDH) was used as a protein loading marker and has a molecular mass of approximately $37 \mathrm{kDa}$.

presence of apoptotic neutrophils (Figure 3). The diameters of monocultured myotubes decreased by approximately $15 \%$ after 1 day and by $20 \%$ after 2 days in low serum medium, whereas the addition of macrophages containing apoptotic neutrophils completely prevented the atrophic process (Figure 4A). Myotube length was unchanged between the different groups at every time point (Figure 4B). The protein content of monocultured myotubes decreased by $10 \%$ after 2 days in low serum medium, whereas the presence of anti-inflammatory macrophages prevented this decrease (Figure 5). Furthermore, the myosin heavy chain content of macro-

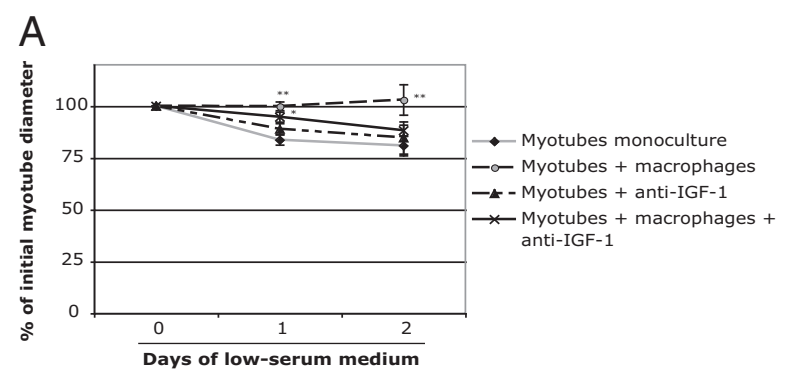

\section{B}

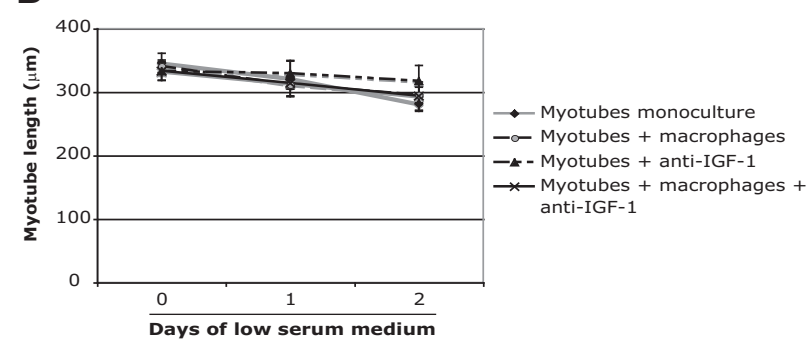

Figure 4. A: Myotube diameters and length in mono- and cocultures after low serum medium-induced myotube atrophy and IGF-1 neutralization. Myotube diameters (A) and length (B) were measured in myotube monocultures, myotubes cocultured with macrophages, myotubes incubated with anti-IGF-1, and myotubes cocultured with macrophages and anti-IGF-1. Values are expressed as means $\pm \operatorname{SEM}(n=12$ on day 1 and $n=6$ on day 2$)$. Significantly different from myotube monocultures, ${ }^{*} P<0.05$. Significantly different from myotubes + anti-IGF-1, ${ }^{* *} P<0.05$. No significant difference between the different groups was observed for myotube length.

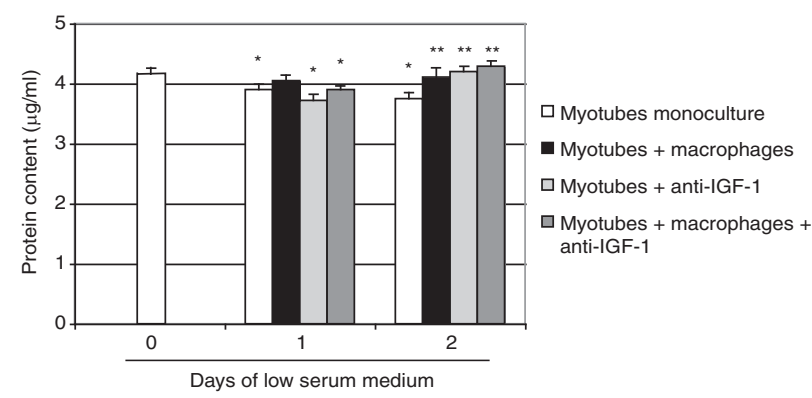

Figure 5. Protein content of myotubes. Protein content was measured on day 0 and after 1 and 2 days of atrophy in myotube monocultures, myotubes cocultured with macrophages, myotubes incubated with anti-IGF-1, and myotubes cocultured with macrophages and anti-IGF-1. Values are expressed as means $\pm \operatorname{SEM}$ ( $n=6$ for every group at every time point). Significantly different from the protein content before atrophy, ${ }^{*} P<0.05$. Significantly different from myotube monocultures, ${ }^{* *} P<0.05$.

phage/myotube cocultures had increased by $10 \%$ after 1 day in low serum medium but had decreased by $10 \%$ in the myotube monocultures (Figure 6, A and B). This difference disappeared after 2 days.

To assess the involvement of IGF-1 released by macrophages on myotube growth, an anti-IGF-1 antibody was added to the coculture system. Anti-IGF-1 inhibited the protective effect of macrophages on myotube diameter in the macrophage/myotube cocultures but had no effect on monocultured myotubes. More importantly, the anti-IGF-1 antibody reduced the myosin heavy chain content of the coculture system by $15 \%$ after 2 days but had no effect on the myosin heavy chain content of

A

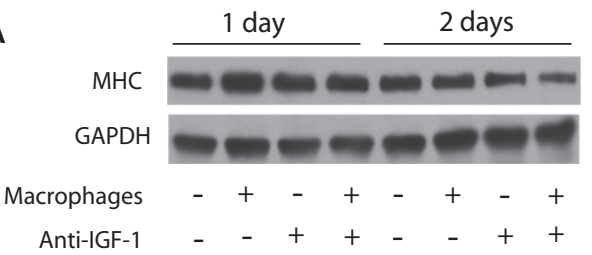

$\mathrm{B}$

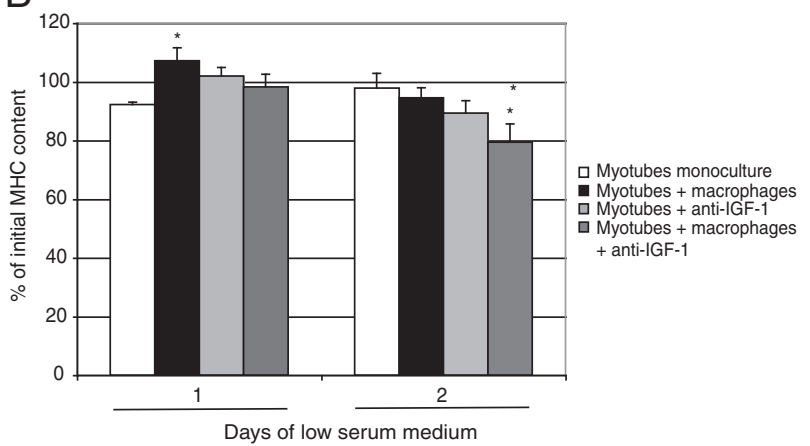

Figure 6. Myosin heavy chain (MHC) content of mono- and cocultures after low serum medium-induced myotube atrophy and IGF-1 neutralization. The myosin heavy chain content was quantified in myotube monocultures, myotubes cocultured with macrophages, myotubes incubated with anti-IGF-1, and myotubes cocultured with macrophages and anti-IGF-1. A: The myosin heavy chain has a molecular mass of approximately $200 \mathrm{kDa}$. B: Glyceraldehyde-3-phosphate dehydrogenase (GAPDH) was used as a protein loading marker and has a molecular mass of approximately $37 \mathrm{kDa}$. Band intensities were quantified in triplicate and were expressed as means $(n=6)$ for each experiment. Significantly different from myotube monocultures, ${ }^{*} P<0.05$. Significantly different from myotubes cocultured with macrophages, ${ }^{* * *} P<0.05$. 
monocultured myotubes. Lastly, anti-IGF-1 did not affect the protein content of monocultured myotubes or macrophage/myotube cocultures at day 1 , but increased the protein content of myotubes monocultured and cocultured at day 2 .

\section{Discussion}

Macrophages play various roles in tissue injury, repair, and growth. ${ }^{27-29}$ The large number of macrophages at sites of injury during the resolution of the inflammatory reaction and the beginning of the repair phase and their ability to switch from a proinflammatory to an anti-inflammatory phenotype make them an excellent candidate for the promotion of muscle repair and growth. ${ }^{30,31}$ In the present article, we demonstrated that macrophages play a critical role in muscle force recovery after muscle atrophy. We also showed, using a macrophage/myotube coculture system, that the IGF-1 released by macrophages protects against myotube atrophy and the decrease in myosin heavy chain content.

Hindlimb unloading/reloading very quickly induces significant muscle atrophy associated with a classic inflammatory reaction. ${ }^{15,32}$ The effect of etoposide on circulating leukocytes that we reported is in agreement with the observations of Van't Wout et al in $1989,{ }^{18}$ who described an $82 \%$ decrease in circulating monocytes and a small but nonsignificant decrease in blood granulocytes after 3 days of etoposide injections. Daily etoposide injections induced a similar reduction in macrophages in reloaded soleus muscles at day 3 . However, although the macrophage count in the blood remained low, the macrophage count in the soleus muscles reached placebo levels on day 7 after reloading. The increase in muscle macrophage counts at later time points can be explained by either the slow recruitment of remaining monocytes into the circulation or the proliferation of newly recruited or endogenous macrophages to the site of injury. This latter possibility is supported by the fact that local proliferation of resident macrophages has been observed in denervated skeletal muscle. ${ }^{33}$ The neutrophil content also rose, increasing 2 -fold by day 7 after reloading in soleus muscles from mice injected with etoposide relative to matched placebo mice. Because macrophages can act as major scavengers for cell debris, the increase in neutrophils may be due to a decrease in macrophage content, which would reduce the clearance of apoptotic neutrophils. ${ }^{34}$ An alternative possibility is that neutrophils are massively recruited to compensate for the lack of muscle macrophages at earlier times in etoposide-injected mice. ${ }^{35}$ Complex immunological adaptations thus occur in skeletal muscle to circumvent the generalized deficiency in circulating monocytes.

We also showed that etoposide induced a 25 to $30 \%$ reduction in maximum tetanic tension in soleus muscles on days 7 and 14 after reloading. The increase in the neutrophil content of soleus muscles from etoposideinjected mice is probably not responsible for the delay in the improvement of muscle force production on days 7 and 14 of reloading because we reported previously that the presence of neutrophils in reloaded soleus muscles did not influence muscle recovery and function. Indeed, we observed no difference in muscle force recovery between mice depleted or not in neutrophils at any time point of reloading. ${ }^{14}$ Thus, the results presented here indicate that macrophages act as a key player in muscle regrowth from atrophy. The delay between macrophage depletion observed at day 3 and muscle force impairment first detected at day 7 may be attributed to the importance of macrophages in initiating muscle recovery. Macrophages have been shown in vitro to stimulate satellite cell migration and myoblast proliferation and to promote fusion into myotubes. ${ }^{36,37}$ Perturbation of these initial steps of muscle healing will subsequently have an important impact on fiber maturation and muscle repair later during the recovery process. The importance of macrophages in muscle repair and growth is also supported by a number of in vivo investigations. For example, the injection of macrophage-conditioned medium directly into injured muscles accelerates the recovery of fiber size and total muscle mass. ${ }^{38}$ Furthermore, macrophage depletion models after muscle freeze injury, muscle graft surgery, or modified mechanical loading showed that in the absence of macrophages, myofibers remain in the necrotic state, the number of regenerating and centronucleated myofibers is reduced, and muscle regrowth is impaired. ${ }^{39-41}$ Our results and those of others indicate that macrophages play a major role in muscle recovery and that the presence of macrophages in sufficient numbers during the critical inflammation resolution and muscle recovery initiation phase is essential for the fast and complete recovery of atrophied muscles.

We cultured macrophages with apoptotic neutrophils and then added them to cultured myotubes to more accurately mimic the inflammatory environment to which atrophied muscles are exposed during muscle regrowth. Our results revealed a major decrease in COX-2 expression by macrophages, indicating that coculturing them with apoptotic neutrophils favors their conversion to an anti-inflammatory phenotype. Previous coculture experiments have also confirmed that the phagocytosis of neutrophils by macrophages and the release of specific cytokines by neutrophils can induce macrophages to switch from a proinflammatory to an anti-inflammatory phenotype. ${ }^{11,42}$ The present results clearly demonstrated that the presence of macrophages expressing an antiinflammatory profile completely prevents the decrease in myotube size, protein content, and myosin heavy chain content. The positive effects of macrophages on muscle growth may be caused by direct contact between macrophages and myotubes and/or by the release of anabolic growth factors by macrophages. Supporting the first hypothesis, cell-cell interactions between macrophages and endothelial cells stimulate endothelial cell proliferation and migration. ${ }^{43}$ Moreover, the release of cytokines by macrophages is partly regulated in a contact-dependent manner. ${ }^{44}$ Macrophages also release mitogenic growth factors for myogenic cells and establish cell-cell interactions that protect myogenic cells from apoptosis. ${ }^{36,45,46} \mathrm{IL}-4$, IL-6, fibroblast growth factor, and IGF-1 as well as several other cytokines and growth 
factors influence myogenic cell behavior. For example, IL-6 stimulates satellite cell proliferation and muscle growth, ${ }^{47,48}$ whereas IL-4 has a fusogenic effect on myogenic cells. ${ }^{49}$ Indeed, the neutralization of IL-4 and the use of myotubes deficient in IL-4 reduce myoblast fusion and consequently the size of myotubes. ${ }^{49,50}$ Fibroblast growth factor and IGF-1 also have a strong ability to stimulate myoblast proliferation and differentiation, respectively. ${ }^{51}$ The positive effect of macrophages on muscle regrowth is thus mediated by multiple and complex mechanisms that remain to be investigated.

The present findings showed that anti-IGF-1 inhibits the protective effect that macrophages have on myotube size and myosin heavy chain content, indicating that that IGF-1 is released by macrophages and acts directly on myotube growth. Consistent with these results, a recent study showed that there is a correlation between the level of IGF-1 mRNA expression and the peak of macrophage accumulation in mouse soleus muscles after unloading and reloading. ${ }^{52} \mathrm{~A}$ relationship between IGF-1 and macrophages has also been corroborated after muscle freeze injury, in which macrophage depletion is associated with a major decrease in IGF-1 mRNA and impairment in muscle repair. ${ }^{40}$ Additional support for a role for IGF-1 comes from transgenic mice in which local expression of IGF-1 strongly accelerates the recovery of muscle force after muscle cardiotoxin injury. ${ }^{53}$ The positive effect of IGF-1 on muscle growth is mediated either by an increase in protein synthesis and/or a decrease in protein degradation. IGF-1 is a powerful activator of the Akt pathway, which stimulates the anabolic downstream effector mammalian target of rapamycin (mTOR) and inhibits protein synthesis repressors such as glycogen synthase kinase 3 (GSK-3 $\beta)^{54,55}$ The phosphorylation of Akt by IGF-1 can also inhibit protein degradation by inactivating the Forkhead box $\mathrm{O}$ (FOXO), which leads to the inhibition of the apoptotic and degradative effectors atrogin-1 and muscle ring finger-1 (MuRF-1). ${ }^{54,56}$ Surprisingly, unlike the decrease in myotube diameter and myosin heavy chain content, the neutralization of IGF- 1 in the coculture system resulted in an increase in protein content after 2 days. These results suggested that the effect of macrophages, acting via IGF-1, on myotubes is possibly limited to specific proteins such as the contractile protein of the myosin heavy chain. A possible explanation for this distinctive effect of IGF-1 on specific contractile proteins is that it is a weak stimulator of myoblast proliferation and a powerful stimulator of myoblast differentiation. ${ }^{51}$ Anti-IGF-1 may thus decrease myotube formation and increase myoblast proliferation, thereby reducing myotube diameter and myosin heavy chain content while increasing total protein content. These results indicate that IGF-1 is a major component of the mechanism that enables macrophages to protect myotubes from atrophy.

The present findings add to the increasing number of studies demonstrating that macrophages have beneficial effects on muscle recovery from various injuries and disuses. ${ }^{27,51,57-59}$ We showed for the first time that macrophages play a critical role at the functional level in muscle recovery from atrophy. We also demonstrated, using a coculture system, that IGF-1 is partially responsible for the ability of macrophages to protect myotubes from atrophy. It will be interesting to determine the role of other factors involved in this positive effect. Lastly, the present results also suggest indirectly that anti-inflammatory drug prescriptions should be used with circumspection because of the positive effects of various macrophage subpopulations during muscle repair and growth.

\section{References}

1. van Furth R: Monocyte production during inflammation. Comp Immunol Microbiol Infect Dis 1985, 8:205-211

2. De Filippo K, Henderson RB, Laschinger M, Hogg N: Neutrophil chemokines $\mathrm{KC}$ and macrophage-inflammatory protein-2 are newly synthesized by tissue macrophages using distinct TLR signaling pathways. J Immunol 2008, 180:4308-4315

3. Faccioli LH, Souza GE, Cunha FQ, Poole S, Ferreira SH: Recombinant interleukin-1 and tumor necrosis factor induce neutrophil migration "in vivo" by indirect mechanisms. Agents Actions 1990, 30:344-349

4. Frangogiannis NG, Mendoza LH, Lindsey ML, Ballantyne CM, Michael LH, Smith CW, Entman ML: IL-10 is induced in the reperfused myocardium and may modulate the reaction to injury. J Immunol 2000, 165:2798-2808

5. Nathan CF: Secretory products of macrophages. J Clin Invest 1987, 79:319-326

6. Sunderkötter C, Goebeler M, Schulze-Osthoff K, Bhardwaj R, Sorg C Macrophage-derived angiogenesis factors. Pharmacol Ther 1991 51:195-216

7. Bogdan C, Nathan C: Modulation of macrophage function by transforming growth factor $\beta$, interleukin-4, and interleukin-10. Ann NY Acad Sci 1993, 685:713-739

8. Gordon S: Alternative activation of macrophages. Nat Rev Immunol 2003, 3:23-35

9. Stout RD, Suttles J: Functional plasticity of macrophages: reversible adaptation to changing microenvironments. J Leukoc Biol 2004, 76:509-513

10. Lapointe BM, Frenette J, Cote $\mathrm{CH}$ : Lengthening contraction-induced inflammation is linked to secondary damage but devoid of neutrophil invasion. J Appl Physiol 2002, 92:1995-2004

11. Fadok VA, Bratton DL, Konowal A, Freed PW, Westcott JY, Henson PM: Macrophages that have ingested apoptotic cells in vitro inhibit proinflammatory cytokine production through autocrine/paracrine mechanisms involving TGF- $\beta$, PGE2, and PAF. J Clin Invest 1998, 101:890-898

12. Arnold L, Henry A, Poron F, Baba-Amer Y, van Rooijen N, Plonquet A, Gherardi RK, Chazaud B: Inflammatory monocytes recruited after skeletal muscle injury switch into antiinflammatory macrophages to support myogenesis. J Exp Med 2007, 204:1057-1069

13. Chazaud B, Brigitte M, Yacoub-Youssef H, Arnold L, Gherardi R, Sonnet C, Lafuste P, Chretien F: Dual and beneficial roles of macrophages during skeletal muscle regeneration. Exerc Sport Sci Rev 2009, 37:18-22

14. Dumont N, Bouchard P, Frenette J: Neutrophil-induced skeletal muscle damage: a calculated and controlled response following hindlimb unloading and reloading. Am J Physiol Regul Integr Comp Physiol 2008, 295:R1831-R1838

15. Frenette J, St-Pierre M, Cote $\mathrm{CH}$, Mylona E, Pizza FX: Muscle impairment occurs rapidly and precedes inflammatory cell accumulation after mechanical loading. Am J Physiol Regul Integr Comp Physiol 2002, 282:R351-R357

16. Tidball JG, Berchenko E, Frenette J: Macrophage invasion does not contribute to muscle membrane injury during inflammation. J Leukoc Biol 1999, 65:492-498

17. Morey-Holton ER, Globus RK: Hindlimb unloading rodent model: technical aspects. J Appl Physiol 2002, 92:1367-1377

18. van't Wout JW, Linde I, Leijh PC, van Furth R: Effect of irradiation, cyclophosphamide, and etoposide (VP-16) on number of peripheral blood and peritoneal leukocytes in mice under normal conditions and during acute inflammatory reaction. Inflammation 1989, 13:1-14

19. Lapointe BM, Cote $\mathrm{CH}$ : Anesthetics can alter subsequent in vitro 
assessment of contractility in slow and fast skeletal muscles of rat. Am J Physiol 1999, 277:R917-R921

20. Côté $\mathrm{CH}$, Perreault $\mathrm{G}$, Frenette J: Carbohydrate utilization in rat soleus muscle is influenced by carbonic anhydrase III activity. Am J Physiol 1997, 273:R1211-R1218

21. McDonald PP, Fadok VA, Bratton D, Henson PM: Transcriptional and translational regulation of inflammatory mediator production by endogenous TGF- $\beta$ in macrophages that have ingested apoptotic cells. J Immunol 1999, 163:6164-6172

22. Ralph P, Nakoinz I: Phagocytosis and cytolysis by a macrophage tumour and its cloned cell line. Nature 1975, 257:393-394

23. Yaffe D, Saxel O: Serial passaging and differentiation of myogenic cells isolated from dystrophic mouse muscle. Nature 1977, 270: 725-727

24. Radi ZA: Pathophysiology of cyclooxygenase inhibition in animal models. Toxicol Pathol 2009, 37:34-46

25. Seibert K, Zhang Y, Leahy K, Hauser S, Masferrer J, Perkins W, Lee $L$, Isakson P: Pharmacological and biochemical demonstration of the role of cyclooxygenase 2 in inflammation and pain. Proc Natl Acad Sci USA 1994, 91:12013-12017

26. Tsatsanis C, Androulidaki A, Venihaki M, Margioris AN: Signalling networks regulating cyclooxygenase-2. Int J Biochem Cell Biol 2006, 38:1654-1661

27. Duffield JS, Forbes SJ, Constandinou CM, Clay S, Partolina M, Vuthoori S, Wu S, Lang R, Iredale JP: Selective depletion of macrophages reveals distinct, opposing roles during liver injury and repair. J Clin Invest 2005, 115:56-65

28. Pollard JW: Trophic macrophages in development and disease. Nat Rev Immunol 2009, 9:259-270

29. Robertson TA, Grounds MD, Papadimitriou JM: Elucidation of aspects of murine skeletal muscle regeneration using local and whole body irradiation. J Anat 1992, 181(Pt 2):265-276

30. Mosser DM, Zhang X: Activation of murine macrophages. Curr Protoc Immunol 2008, Chapter 14:Unit 14.12

31. Smith C, Kruger MJ, Smith RM, Myburgh KH: The inflammatory response to skeletal muscle injury: illuminating complexities. Sports Med 2008, 38:947-969

32. Allen DL, Bandstra ER, Harrison BC, Thorng S, Stodieck LS, Kostenuik PJ, Morony S, Lacey DL, Hammond TG, Leinwand LL, Argraves WS, Bateman TA, Barth JL: Effects of spaceflight on murine skeletal muscle gene expression. J Appl Physiol 2009, 106:582-595

33. Murray MA, Robbins N: Cell proliferation in denervated muscle: identity and origin of dividing cells. Neuroscience 1982, 7:1823-1833

34. Weigert A, Jennewein C, Brune B: The liaison between apoptotic cells and macrophages-the end programs the beginning. Biol Chem 2009, 390:379-390

35. Dumont N, Lepage K, Cote CH, Frenette J: Mast cells can modulate leukocyte accumulation and skeletal muscle function following hindlimb unloading. J Appl Physiol 2007, 103:97-104

36. Cantini M, Massimino ML, Bruson A, Catani C, Dalla Libera L, Carraro $U$ : Macrophages regulate proliferation and differentiation of satellite cells. Biochem Biophys Res Commun 1994, 202:1688-1696

37. Robertson TA, Maley MA, Grounds MD, Papadimitriou JM: The role of macrophages in skeletal muscle regeneration with particular reference to chemotaxis. Exp Cell Res 1993, 207:321-331

38. Cantini M, Giurisato E, Radu C, Tiozzo S, Pampinella F, Senigaglia D, Zaniolo G, Mazzoleni F, Vitiello L: Macrophage-secreted myogenic factors: a promising tool for greatly enhancing the proliferative capacity of myoblasts in vitro and in vivo. Neurol Sci 2002, 23:189-194

39. Grounds MD, Davies M, Torrisi J, Shavlakadze T, White J, Hodgetts S: Silencing TNF $\alpha$ activity by using Remicade or Enbrel blocks inflammation in whole muscle grafts: an in vivo bioassay to assess the efficacy of anti-cytokine drugs in mice. Cell Tissue Res 2005, 320: $509-515$

40. Summan M, Warren GL, Mercer RR, Chapman R, Hulderman T, Van Rooijen N, Simeonova PP: Macrophages and skeletal muscle regeneration: a clodronate-containing liposome depletion study. Am J Physiol Regul Integr Comp Physiol 2006, 290:R1488-R1495

41. Tidball JG, Wehling-Henricks M: Macrophages promote muscle membrane repair and muscle fibre growth and regeneration during modified muscle loading in mice in vivo. J Physiol 2007, 578:327-336
42. Daley JM, Reichner JS, Mahoney EJ, Manfield L, Henry WL Jr, Mastrofrancesco B, Albina JE: Modulation of macrophage phenotype by soluble product(s) released from neutrophils. J Immunol 2005, 174:2265-2272

43. Schubert SY, Benarroch A, Ostvang J, Edelman ER: Regulation of endothelial cell proliferation by primary monocytes. Arterioscler Thromb Vasc Biol 2008, 28:97-104

44. Brennan F, Foey A: Cytokine regulation in RA synovial tissue: role of T cell/macrophage contact-dependent interactions. Arthritis Res 2002, 4(Suppl 3):S177-S182

45. Massimino ML, Rapizzi E, Cantini M, Libera LD, Mazzoleni F, Arslan $\mathrm{P}$, Carraro U: ED2 ${ }^{+}$macrophages increase selectively myoblast proliferation in muscle cultures. Biochem Biophys Res Commun 1997, 235:754-759

46. Sonnet C, Lafuste P, Arnold L, Brigitte M, Poron F, Authier FJ, Chretien F, Gherardi RK, Chazaud B: Human macrophages rescue myoblasts and myotubes from apoptosis through a set of adhesion molecular systems. J Cell Sci 2006, 119:2497-2507

47. Cantini M, Massimino ML, Rapizzi E, Rossini K, Catani C, Dalla Libera $\mathrm{L}$, Carraro U: Human satellite cell proliferation in vitro is regulated by autocrine secretion of IL-6 stimulated by a soluble factor(s) released by activated monocytes. Biochem Biophys Res Commun 1995, 216:49-53

48. Serrano AL, Baeza-Raja B, Perdiguero E, Jardi M, Munoz-Canoves P: Interleukin-6 is an essential regulator of satellite cell-mediated skeletal muscle hypertrophy. Cell Metab 2008, 7:33-44

49. Horsley V, Pavlath GK: Forming a multinucleated cell: molecules that regulate myoblast fusion. Cells Tissues Organs 2004, 176:67-78

50. Horsley V, Jansen KM, Mills ST, Pavlath GK: IL-4 acts as a myoblast recruitment factor during mammalian muscle growth. Cell 2003, 113:483-494

51. Allen RE, Boxhorn LK: Regulation of skeletal muscle satellite cell proliferation and differentiation by transforming growth factor- $\beta$, insulin-like growth factor I, and fibroblast growth factor. J Cell Physiol 1989, 138:311-315

52. Heinemeier KM, Olesen JL, Haddad F, Schjerling P, Baldwin KM, Kjaer M: Effect of unloading followed by reloading on expression of collagen and related growth factors in rat tendon and muscle. J Appl Physiol 2009, 106:178-186

53. Pelosi L, Giacinti C, Nardis C, Borsellino G, Rizzuto E, Nicoletti C, Wannenes F, Battistini L, Rosenthal N, Molinaro M, Musaro A: Local expression of IGF-1 accelerates muscle regeneration by rapidly modulating inflammatory cytokines and chemokines. FASEB J 2007, 21:1393-1402

54. Dehoux M, Gobier C, Lause P, Bertrand L, Ketelslegers JM, Thissen JP: IGF-I does not prevent myotube atrophy caused by proinflammatory cytokines despite activation of Akt/Foxo and GSK-3 $\beta$ pathways and inhibition of atrogin-1 mRNA. Am J Physiol Endocrinol Metab 2007, 292:E145-E150

55. Nader GA, McLoughlin TJ, Esser KA: mTOR function in skeletal muscle hypertrophy: increased ribosomal RNA via cell cycle regulators. Am J Physiol Cell Physiol 2005, 289:C1457-C1465

56. Nader GA: Molecular determinants of skeletal muscle mass: getting the "AKT" together. Int J Biochem Cell Biol 2005, 37:1985-1996

57. Goren I, Allmann N, Yogev N, Schurmann C, Linke A, Holdener M, Waisman A, Pfeilschifter J, Frank S: A transgenic mouse model of inducible macrophage depletion: effects of diphtheria toxin-driven lysozyme M-specific cell lineage ablation on wound inflammatory, angiogenic, and contractive processes. Am J Pathol 2009, 175: 132-147

58. Shechter R, London A, Varol C, Raposo C, Cusimano M, Yovel G, Rolls A, Mack M, Pluchino S, Martino G, Jung S, Schwartz M: Infiltrating blood-derived macrophages are vital cells playing an antiinflammatory role in recovery from spinal cord injury in mice, PLoS Med 2009, 6:e1000113

59. van Amerongen MJ, Harmsen MC, van Rooijen $\mathrm{N}$, Petersen $\mathrm{AH}$, van Luyn MJ: Macrophage depletion impairs wound healing and increases left ventricular remodeling after myocardial injury in mice. Am J Pathol 2007, 170:818-829 